\title{
7. REFERÊNCIAS
}

AGUIAR, M. O. e MENDONÇA, M. S. Morfo-anatomia da semente de Euterpe precatoria Mart. (Palmae). Revista Brasileira de Sementes. Pelotas: 2003. Disponível em: <http://www.scielo.br/scielo.php?pi$\mathrm{d}=$ S0101-31222003000100007\&script=sci_arttext $>$ Acesso em: 19/07/2013.

ALUN-JONES, Deborah e AYTON, Jonh. Charming: the magic of charm jewelry. UK: Thames et Hudson, 2005.

ANDERSOM, W. R. Malpighiaceae. Botany of the Guiana Highland. Disponível em: <https://www.lsa.umich.edu/herb/malpigh/MALPpdf/FlDucke.pdf> Acesso em: 20/07/2013.

ARAÚJO, Maria Gracimar Pachedo de, LEITÃO, Antônio Machado e MENDONÇA, Maria Silvia de. Morfologia do fruto e da semente de inajá (Attalea maripa (Aubl.) Mart.) - PALMAE. Revista Brasileira de Sementes, vol. 22, n 2, p. 31-38, 2000.

BANDEIRA, Julio. Sementes ornamentais do Brasil. Rio de Janeiro: Reler, 2008.

BENATTI, L. P. Aspectos sustentáveis na cadeia de valor da biojoia: design aplicado a produtos artesanais. Anais do $4^{\circ}$ Simpósio Paranaense de Design Sustentável. Curitiba: UFPR, 2012.

BENATTI, L. P. e SILVA, A. C. M. Influence of indigenous culture in the use of ornamental seeds as fashion accessories: how design can make the segment of biojewel more competitive. 1st International Fashion and design congress. Guimarães: 2012.

BONSIEPE, G. Design: do material ao digital. Florianópolis: FIESC/IEL, 1997.

BORGES, A. Maurício Azeredo: a construção da identidade brasileira no mobiliário. São Paulo: Instituto Lina Bo e P. M. Bardi, 1999.

BUENO, Eduardo. Brasil: uma história: cinco séculos de um país em construção. São Paulo: Leya, 2010.

CARVALHO, Mônica. Portal da designer de biojoias. Disponível em: <http:// www.monicacarvalho.com.br/> Acesso em: 20 de setembro de 2012.

CARVALHO, Paulo Ernani Ramalho. Ocorrência natural in Embrapa Florestas. Disponível em: <http://www.agencia.cnptia.embrapa.br/gestor/especies_arboreas_brasileiras/arvore/CONT000fuo1d7rz02wyiv807nyi6sfhxiys2.html> Acesso em: 20 de abril de 2013.

CAVAZZA, N. Psicologia das atitudes e opiniões. São Paulo: Edições Loyola, 2005 . 
CEAP DESIGN. Centro de Estudos Ambientais e Paisagísticos. Disponível em: <http://www.ceapdesign.com.br/familias_botanicas/poaceae.html > Acesso em: 20/07/2013.

COY, Martin e KOLHLEPPP, Gerd (coord.). Amazônia sustentável: desenvolvimento sustentável entre políticas públicas, estratégias inovadoras e experiências locais. Rio de Janeiro : Garamond; Tübinger, Alemanha: Geographischen Instituts der Universität Tübingen, 2005.

DEBRET, Jean-Baptiste. Caderno de viagem. Texto e organização: Julio Bandeira. Rio de Janeiro: Sextante, 2006.

DESIGN, Brasil faz. Brasil faz design $6^{a}$ edição. Curitiba, BFD 2004.

EMBRAPA AMAZÔNIA ORIENTAL. Produção e montagem de biojoias com sementes da Amazônia. Dia de campo na TV, 2006.

FEEVALE. Materioteca. Disponível em: <http://materioteca.feevale. br:8080/webmaterioteca/externo/index.jsp> Acesso em: 10/04/2012.

FELIX, Ana Angélica Alves. Identificação e desenvolvimento de técnica alternativa de controle de fungos em sementes utilizadas no artesanato. Universidade de Brasília, 2007. Disponível em: <http://repositorio.bce.unb. br/bitstream/10482/3322/1/2007_AnaAngelicaAlvesFelix.PDF> Acesso em: 15/03/2012.

FILGUEIRAS, T. S. Coix in Lista de espécies da flora do Brasil. Jardim Botânico do Rio de Janeiro. Disponível em: <http://floradobrasil.jbrj.gov.br/ jabot/listaBrasil/FichaPublicaTaxonUC/FichaPublicaTaxonUC.do?id=FB13126> Acesso em: 16 de abril de 2013.

FORAGES, Tropical. Mucuna pruriens. Disponível em: <http://www.tropicalforages.info/key/Forages/Media/Html/Mucuna_pruriens.htm> Acesso em: 16 de abril de 2013.

GOMES FILHO, João. Gestalt do objeto: sistema de leitura visual da forma. São Paulo: Escrituras Editora, 2000.

GUÍA DE CONSULTA BOTÂNICA II. Facultad de Ciencias Exactas y Naturales y Agrimensura (UNNE). Disponível em: < http://www.biologia.edu.ar/ diversidadv/fascIII/42.\%20Araliaceae.pdf > Acesso em: 20/07/2013.

KAZAZIAN, Thierry. Haverá a idade das coisas leves. São Paulo: Editora Senac São Paulo, 2005.

KRUCKEN, Lia. Design e território: valorização de identidades e produtos locais. São Paulo: Studio Nobel, 2009.

LANA, S. L. B. A complexidade dos métodos em design. Cadernos de estudos avançados em design: métodos. EdUEMG, 2011.

LANA, S. L. B., KRUCKEN, L., SILVA, A. C. M., BENATTI, L. P. Design de biojoias: desenvolvimento de produtos com perfil sustentável. VI Encontro Nacional da ANPPAS. Belém, 2012. 
LANA, S. L. B. e BENATTI, L. P. Innovation in the techniques of decorative finish on Brazilian ornamental seeds: design applied to products with sustainable profile. 4th International Forum of Design as a Process. Belo Horizonte: UEMG, 2012.

LANA, S. L. B. e BENATTI, L. P. Potencialidades do uso de sementes ornamentais na moda: Uma visão panorâmica da biojoia brasileira. Anais do X Congresso Brasileiro de Pesquisa e Desenvolvimento em Design. São Luís: EDUFMA, 2012.

LANA, S. L. B. e BENATTI, L. P. Usinagem aplicada à sementes ornamentais brasileiras: utilizando o design para tornar o setor da biojoia mais competitivo. Projética: revista científica de design. Londrina: UEL, 2012.

LIMA, H. C. Hymenaea in Lista de Espécies da Flora do Brasil. Jardim Botânico do Rio de Janeiro. Disponível em: <http://floradobrasil.jbrj.gov.br/jabot/ floradobrasil/FB22972> Acesso em: 20 de abril de 2013.

LORENZI, Harri. Árvores brasileiras: manual de identificação e cultivo de plantas arbóreas do Brasil, vol. 1. Nova Odessa, SP: Instituto Plantarum, 2008.

LORENZI, Harri. Árvores brasileiras: manual de identificação e cultivo de plantas arbóreas do nativas do Brasil, vol. 2. Nova Odessa, SP: Instituto Plantarum, 2009.

LORENZI, Harri. Árvores brasileiras: manual de identificação e cultivo de plantas arbóreas nativas do Brasil, vol 3. Nova Odessa, SP: Instituto Plantarum, 2009.

LORENZI, Harri [et al.]. Flora brasileira Lorenzi: Arecaceae (palmeiras). Nova Odessa, SP: Instituto Plantarumm, 2010.

MAMEDE, M. C. H. Byrsonima in Lista de Espécies da Flora do Brasil. Jardim Botânico do Rio de Janeiro. Disponível em: < http://floradobrasil.jbrj.gov. br/jabot/floradobrasil/FB8827> Acesso em: 20 de abril de 2013.

MANZINI, Ezio e VEZZOLI, Carlo. O Desenvolvimento de produtos sustentáveis. São Paulo: Editora da Universidade de São Paulo, 2005.

MCGRATH, Jinks. Acabados decorativos en joeyría: del esmaltado y el grabado a la incrustación y el granulado. Barcelona: Promopress, 2007.

MOURA, T. M. e TOZZI, A. M. G. A. Mucuna in Lista de Espécies da Flora do Brasil. Jardim Botânico do Rio de Janeiro. Disponível em: <http://floradobrasil.jbrj.gov.br/jabot/floradobrasil/FB83489> Acesso em: 20 de abril de 2013.

MUNDO DOS ÓLEOS. Óleo de eucalipto citriodora. Disponível em: $<$ http://www.mundodosoleos.com/oleo-de-eucalipto-citriodora-1litro> Acesso em 21 de abril de 2013.

NATURAL JOIAS. Portal de empresa de venda de sementes ornamentais. Disponível em: <https://www.naturaljoias.com.br/quem_somos.php> Acesso em: 10/09/2012. 
NOSSAS ÁRVORES. Portal de espécies apadrinhadas. Disponível em: <https://arvores.greennation.com.br/species/92 > Acesso em: 02/03/2017.

PEZZOLO, Dinah Bueno. Por dentro da moda: definições e experiências. São Paulo: Editora Senac São Paulo,2009.

OKAMOTTO, Paulo. Artesanato é negócio. Artesanato: um negócio genuinamente brasileiro. Sebrae, volume 1, número 1, março de 2008.

OLIVEIRA, Maria do Socorro Padilha de; NETO, João Tomé de Farias e PENA, Rosinelson da Silva. Açaí: técnicas de cultivo e processamento - Fortaleza: Instituto Frutal, 2007.

RAINTREE NUTRITION. Velvet Bean (Mucuna pruriens). Tropical plant database. Disponível em: <http://rain-tree.com/velvetbean.htm\#.UW2vwrWG1hE> Acesso em: 26 de abril de 2013.

RAMO JOALHEIRO. Jóias ecológicas brasileiras são sucesso no exterior. Disponível em: <http://www.ramojoalheiro.com.br/noticias/interna.asp?cod=568> Acesso em: 21/07/2010

RIBEIRO. Berta G. Dicionário do artesanato indígena. São Paulo: Editora da Universidade de São Paulo, 1988.

SALEM, Vidal. Tingimento têxtil: fibras, conceitos e tecnologias. São Paulo: Blucher: Golden Tecnologia, 2010.

SEBRAE. Programa Sebrae de Artesanato. Termo de referência: março de 2004.

SILVA, André Carvalho Mol e BENATTI, Lia Paletta. Biojoia: Aplicação de Materiais Naturais na Joalheria. Lorena: $5^{\circ}$ Workshop de Design e Materiais, 2010.

VALLE, M. Sementes florestais utilizadas em artesanato no Rio de Janeiro. Disponível em: http://www.if.ufrrj.br/rioesba/monografia/Maria_Joana_Lima_ Valente_do_Valle.pdf Acesso em: 01/09/2011. 